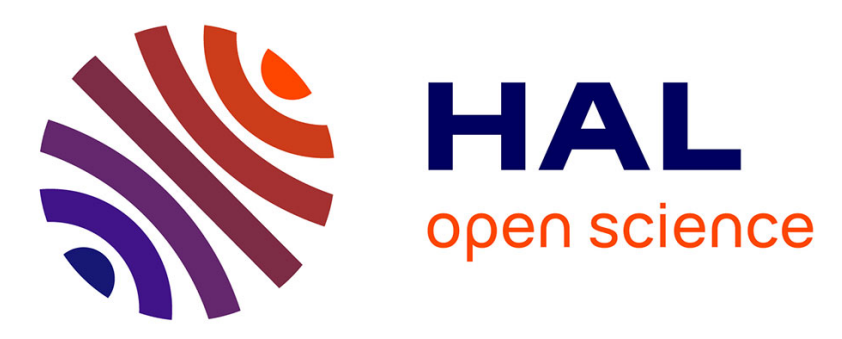

\title{
Embedded Powerline Communication in Large Scale Distribution Automation and Demand Side Management System
}

\author{
Liping Lu, Jianhua Zhou, Jian Hu, Gangyan Li, Ye-Qiong Song
}

\section{- To cite this version:}

Liping Lu, Jianhua Zhou, Jian Hu, Gangyan Li, Ye-Qiong Song. Embedded Powerline Communication in Large Scale Distribution Automation and Demand Side Management System. The 2nd IEEE/ASME International Conference on Mechatronic and Embedded Systems and Applications, Aug 2006, Beijing, China. inria-00113782

\section{HAL Id: inria-00113782 \\ https://hal.inria.fr/inria-00113782}

Submitted on 14 Nov 2006

HAL is a multi-disciplinary open access archive for the deposit and dissemination of scientific research documents, whether they are published or not. The documents may come from teaching and research institutions in France or abroad, or from public or private research centers.
L'archive ouverte pluridisciplinaire HAL, est destinée au dépôt et à la diffusion de documents scientifiques de niveau recherche, publiés ou non, émanant des établissements d'enseignement et de recherche français ou étrangers, des laboratoires publics ou privés. 


\title{
Embedded Powerline Communication in Large Scale Distribution Automation and Demand Side Management System
}

\author{
LiPing Lu, JianHua Zhou, Jian Hu, GangYan Li, and YeQiong Song
}

\begin{abstract}
Implementation of Distribution Automation (DA) and Demand Side Management (DSM) intended to serve both utilities and a large number of customers which spread over a large geographical area, requires a wide-area two-way communication system. DA and DSM devices (customer meters, switches, etc.), embedded with Powerline communication, can use the powerline as a communication medium, to construct a cost-effective and high speed transmission communication network. REMPLI project aims at designing and implementing a communication infrastructure for distributed data acquisition and remote control operation using the power grid as the communication medium. In this paper, we evaluate the REMPLI PLC network performance to show how the requirements for both DA/DSM applications can be met. And a scheduling policy is proposed to provide the applications with differentiate QoS in a multitasking environment.
\end{abstract}

\section{INTRODUCTION}

$\mathrm{M}$ ODERN society demands a reliable and high quality energy supply. For the distribution utilities, it is substantial to provide power to customers at acceptable reliability level and to serve the customer in better ways. For effectively control the power distribution network, the comprehensive and real-time measurements should be obtained accurately, continuously and reliably. Many distribution automation (DA) systems have been and will be installed in the distribution systems. However, nowadays there are only a limited number of real-time measurements on the distribution systems [5]. The overhead feeder equipments including reclosers, switches and feeder restoration, and distribution transformer are critical items of equipments in power systems to improving the operation of distribution network. They should be monitored and controlled in DA system. The load monitoring and estimation of customers which have been partially implemented in DSM system, can be an important source of information used by the distribution analysis applications. Large scale system implementation of DA/DSM intended for a utility network serving a large customer base (one million or larger) spread over a large geographical area requires a two-way communication system [1].

The construction of private wire network cost much, since

This work was supported in part by the REMPLI Fifth Framework Program of the EC (project identifier NNE5-2001-00825).

LiPing Lu is with both School of Mechanical \& Electronic Engineering, Wuhan University of Technology, P.R. China and LORIA - INPL, France (e-mail: liping.lu@ loria.fr).

JianHua Zhou, Jian Hu and GangYan Li are with School of Mechatronic Engineering, Wuhan University of Technology, 205 Luoshi Road, 430070, Wuhan,Hubei, P.R.China (e-mail: gangyan.li@whut.edu.cn).

YeQiong Song is with LORIA - INPL, Campus Scientifique, B.P. 239, 54506 Vandoeuvre, France (e-mail: song@1oria.fr). the energy metering and control equipments are distributed widely. And there are the difficulties to access to certain equipments using wireless technology because they are often located in closed environments with metallic obstacles (reinforced concrete walls and tubes). It is ideal to embedded powerline communication (PLC) technologies into the devices for using the powerline as a communication medium to construct an economic, secure and reliable communication system, because no new wires are needed for the low cost, the reliable and high transmission speed PLC chips are available for the efficiency, and power line is owned by the distribution utility for the security.

REMPLI (Remote Energy Management over Power Lines and Internet) system use Medium Voltage (MV) and Low Voltage (LV) power grid as communication media to implements wide-area control and monitoring. Since the distances are long in a wide area PLC network, transmitting a packet from a source to a not immediately reachable destination node requires the packet relay of the intermediate nodes (repeaters). Moreover, considering the dynamic topology change and impossible prediction of the powerline attenuation, repeaters cannot be statically configured. A new routing protocol in REMPLI project, called simple frequency network (SFN), has been developed to provide a dynamic repeater scheme. It is important to evaluate the communication network performance within SFN protocol to show if the application timing constraints can be guaranteed.

The communication system for DA/DSM applications is multi-tasking environment, because functions such as automatic remote meter reading, load management, on-request meter reads, and other distribution control and monitoring functions are simultaneously implemented. And DA applications require support of real-time communication. Thus, QoS mechanisms with differentiate QoS services in PLC protocol should be provided to meet the application requirement.

In this paper, a short overview of the basic requirements for DA and DSM communication systems is given in section II. Next a general REMPLI system overview is given in section III. Then we evaluate the performance of REMPLI architecture in terms of the data transfer time and estimate if the DA and DSM communication requirement can be met in section IV. To meet the different delay requirements of the different application traffics within the DA and DSM, section $\mathrm{V}$ proposes a scheduling policy to provide to applications with differentiate QoS. 


\section{REQUIREMENTS For DA/DSM COMMUNiCATION SYSTEM}

The communication infrastructure should meet the following fundamental requirements [1]:

--The communication system is two-way and can handle massive amounts of data and multi-tasking.

--Data throughput and system response times should meet various application requirements.

--The system should allow for network growth and value-added applications.

DA and DSM applications require data transfer between several remote devices and a central location. In the following, time constraints of DA and DSM applications are given.

\section{A. Distribution Automation}

IEEE has defined DA system as a system that enables an electric utility to remotely monitor, coordinate and operate distribution components, in a real-time mode from remote location. Field instrumentation connected to the equipment or feeder being monitored and controlled are interfaced to a remote terminal units (RTU) that allows data manipulation and help in implementing control action in the field. And RTU gathers data from the equipments and transfers it to the remote control center and receives the commands from remote control center to execute. Generally, there are two kinds of DA RTU: microprocessor based substation and pole-top RTU. Most DA RTUs are distributed over the MV power grids. DA system requires a communication system which should support real-time communication between the control center and RTUs.

Here, the DA-specific requirements for communication systems are given [2]:

--System Size: average $75 \mathrm{MV} / \mathrm{LV}$ substations per HV/MV substation transformer [6]

--Response time to command: < approx. $10 \mathrm{sec}$

--Alarm delay: < approx. $30 \mathrm{sec}$

--User telegram size: In average 20 bytes, up to 128 byte should be supported

Response time to a command is specified in terms of the time when the message leaves the sending application to the time when the receiving application gets the message. Alarm delay is the time from the alarm generated by feeder equipments to the time when this alarm information gets back to control center.

\section{B. Demand side management}

DSM applications, which mainly consist of meter reading, load shedding etc., have different requirements. Most outstanding, the real-time requirements are much less rigorous. The classical ripple control systems (classic power line communication technology with low speed rate < $50 \sim 60 \mathrm{bit} / \mathrm{s}$ ) are capable of implementation of kind of DSM applications, such as load shedding, tariff information distribution functions and e.g., through unidirectional transmission. Herein DSM application requirements focus on the metering reading function and its requirements for communication systems are given: --system size: average 114 customer per MV/LV substation; $75 \mathrm{MV} / \mathrm{LV}$ substation per HV/MV substation transformer [6]

--Response time to an individual read: < approx. $30 \mathrm{sec}$ [2]

--Data volume: approx. 20 bytes/meter/read

--Cycle time for reading: Monthly, weekly, half-hourly (depending on regulatory framework). Profiles can store locally in the meter. It is not necessary to have the data available in real-time, but the metering reading data collection should be finished within the cycle time.

Response time to an individual read is specified in terms of the time when the request message ready for transmission to the time when the application gets response message.

\section{REMPli SyStem ARCHITECTURE}

REMPLI system constructs a hierarchical communication structure shown in Fig. 1. The Application is connected with the Access Point through the company's Intranet, based on a TCP/IP network. Between the Access Point and the Nodes, the used network is PLC network. In PLC network, REMPLI Bridge at the $\mathrm{MV} / \mathrm{LV}$ transformer stations, provide transparent data connection between the MV and LV powerlines. Each REMPLI Node connects with one or more RTUs, such as switchgears and meters. For each of the voltage segments, there is the TDMA Master/Slave communication model. The Access Points and the Bridges are Masters for the MV and LV segments, respectively. The Bridges and Nodes are Slaves for the MV and LV segments, respectively. In this master/slave model, only master can initiate packet transfer. A slave can only send a packet back to the master until its master polls it. So in the whole REMPLI network there are as many independent polls as the masters as shown in Fig. 1.

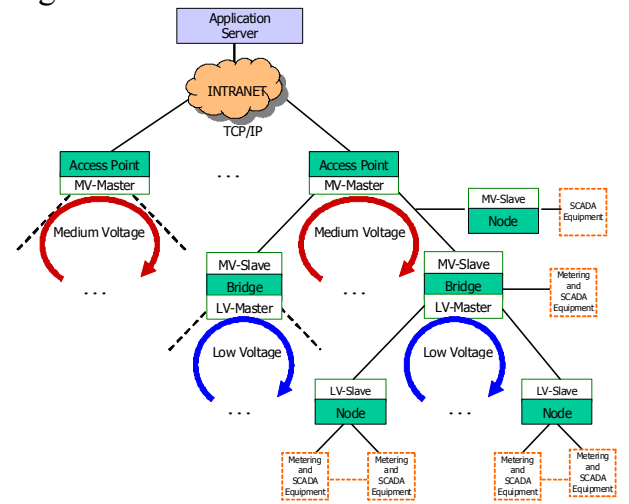

Fig. 1. Typical REMPLI system architecture

We call hereafter autonomous PLC network the piece of PLC network formed by one master and all its slaves. A new flooding-based routing protocol, called SFN protocol [3], has been developed for dynamic repeating the packet. Then, in each autonomous PLC network, all slaves can be potential repeaters, and zero or more repeaters are used to reach the destination according to current powerline situation and transmission distance.

Due to employing new routing technology and combining of the MV and LV power grids, the REMPLI communication 
architecture is different from the traditional PLC network and the communication network performance should be evaluated.

\section{Communication Performance of Rempli System}

Although the REMPLI system performance should include two parts: intranet (TCP/IP) and PLC network, we are only interested in the timing performance of the PLC network, since intranet (TCP/IP) technology is widely used and stable and the time issue is small if intranet uses optical cables.

According to the communication requirements in section II, we define three performance metrics: response time to a command, response time to a request and average polling time. The former two performance metrics are application-to-application delay, which includes the communication processor's process time. In fact, processing times using modern microprocessors are extremely short and can be ignored. So those performance metrics only concerns the communication transmission time. The last performance metrics is to evaluate the periodic tasks such as the cyclic collection of all customer metering reading. Furthermore, in master/slave mode, the slave can not initialize a transmission to master, so the alarm information only can be send back to the master by the cyclic polling.

Since the information transmission can be happened between the Access Point and the MV Node in the MV power grid and between the Access Point and the LV Node in combination with the LV and MV power grids, we evaluate the performance of both the autonomous PLC network and the whole REMPLI PLC network. The performance metrics have different definition and expression formulas.

We do the simulation to evaluate the system performance. The physical layer of powerline communication system is emulated by the Physical Layer Emulator [4] developed by iAd. There are 4 channel models which are Ring_10, Ring_100, Rand_Area Np_100, Rand_Area Np_200. The two former channel models have the topology of ring and the latter two have the topology of a tree with the master as the root and the randomly distributed slaves as leaves. The number in the channel model name indicates the number of the nodes. The upper layer is simulated in OPNET simulation tool.

\section{A. Timing performance in an autonomous PLC network}

In the most general cases, there is a communication failure on one of downlink (master to slave) or uplink (slave to master) channels due to noisy conditions in PLC network. In network layer protocol, a retry mechanism is used, which allows resending a packet after the master did not receive a confirm from the destination slave in a predefine time-out, recovering from the possible loss of packets. Moreover, SFN protocol considers the transmission failure as the signal of the repeater number (called repeater level in the protocol) deficiency. Before each retry begins, the current value of repeater level should be increased one. Therefore, the transmission time of a simple transaction is depended upon the repeater level and retry number.

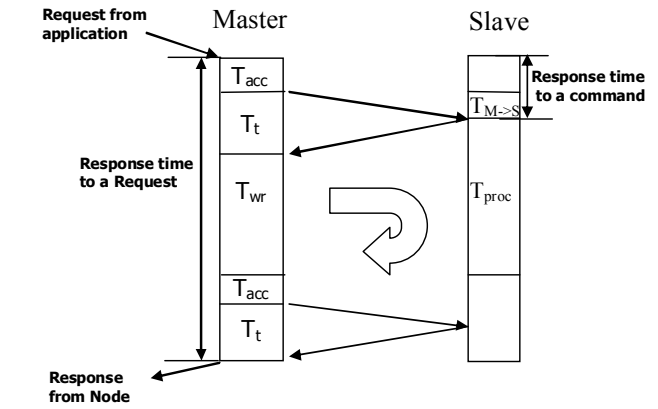

Fig. 2. Schematic representation of application-to-application delay at the Network Layer level in an autonomous PLC network

The response time to a command $\left(T_{c}\right)$ is the duration between the time when the packet is ready for transmission in the master and is arrived at its destination slave $i$ correctly. The total time is:

$$
T_{c}(i)=T_{a c c}+T_{M \rightarrow S}(i)
$$

$T_{a c c}$ means medium access delay. When the packet is ready for send, the channel has been occupied by the transmission of other packets or the high priority packets. The command transmission can't begin until the current transmission tasks have been finished by receiving the confirm from the destination slave or the failure transmission after greater than maximum retry number. So $T_{a c c}$ is a random value and can not be predicted in a multi-tasking environment with aperiodic application functions. In the simulation scenarios, the $T_{a c c}$ can be ignored because we simulate that new command message and request/response message arrive to the master, sequentially after last message has finished the transmission.

$T_{M \rightarrow S}$ represents the transmission duration from sending the command by the master to receiving it correctly by the destination slave. Considering the retry happening, $T_{M \rightarrow S}$ can be calculated by the following formula:

$$
T_{M \rightarrow S}(i)=\left(r_{D L}(i)+n_{\text {retry } i}+1+\sum_{j=0}^{n_{\text {ara }} \sum^{-1}}\left(2 \cdot(j+1)+r_{D L}(i)+r_{U L}(i)\right)\right) \cdot T_{s}
$$

Parameters:

$T_{s} \quad$ duration of one slot for transmitting a packet

$n_{\text {retry } i}$ retry number of node $i$

$r_{D L}(i)$ repeater level of downlink of node $i$ for the fist transmission

$r_{U L}(i)$ repeater level of uplink of node $i$ for the first transmission

The response time to a request $\left(T_{r}\right)$ is the time duration from the request packet ready for transmission to the response packet receiving by the master. The response could be not immediately available upon receiving of the request in the slave, but only after a certain variable delay (typically between $200 \mathrm{~ms}$ to 1.5 seconds, corresponding to the time for a node to get the data from a device, e.g. a meter [6]). For effective utilization of PLC bandwidth, the slave sends a confirm back to the master when it receives a request. The upper layer should give a waiting for response duration $\left(T_{w r}\right)$ to the network layer of the master. In the process time $T_{p o r}$, the response has been prepared and stored in the slave. During $T_{w r}$, the master may commence other transmissions. After 
waiting time of $T_{w r}$, the master will poll the slave again to get the response back. If the master transmits other packets in $T_{w r}$, the $T_{a c c}$ is possible to be required. We assume that one request only generates one packet response from the Node. From Fig. 2, $T_{r}(i)$ is the response time to a request for slave $i$ and the formula to calculate it is given in (3).

$$
T_{r}(i)=T_{a c c}+T_{t}(i)+T_{w r}+T_{a c c}+T_{t}(i)
$$

$T_{t}(i)$ is transmission time from a packet sending by the master to a packet receiving a packet back from the destination slave $i[7]$.

$$
T_{t}(i)=\sum_{j=0}^{n_{\text {retry }} i}\left(2 \cdot(j+1)+r_{D L}(i)+r_{U L}(i)\right) \cdot T_{s}
$$

The average duration of a polling cycle is defined as the time for the master to poll once all the slaves. The formula to calculate it, is given in (5).

$$
D=\sum_{i=2}^{n} T_{t}(i)
$$

Parameters:

$\mathrm{n}$ number of node (master node with $\mathrm{n}=1$ )

TABLE I

RESPONSE TIME TO A COMMAND

\begin{tabular}{cccccc}
\hline \hline $\begin{array}{c}\text { Channel } \\
\text { Model }\end{array}$ & $\begin{array}{c}\text { Maximum } \\
\text { repeater } \\
\text { number }\end{array}$ & $\begin{array}{c}\text { Minimum } \\
\text { repeater } \\
\text { number }\end{array}$ & $\begin{array}{c}\text { Timeslot } \\
\text { duration } \\
(\mathrm{s})\end{array}$ & $\begin{array}{c}\text { Maximum } \\
\text { response } \\
\text { time (s) }\end{array}$ & $\begin{array}{c}\text { Minimum } \\
\text { response } \\
\text { time (s) }\end{array}$ \\
\hline Ring_10 & 2 & 0 & 0.009792 & 0.068544 & 0.009792 \\
Ring_100 & 3 & 0 & 0.009792 & 0.088128 & 0.009792 \\
$\begin{array}{c}\text { RandArea } \\
\text { Np_100 }\end{array}$ & 4 & 0 & 0.009792 & 0.107712 & 0.009792 \\
$\begin{array}{c}\text { RandArea } \\
\text { Np_200 }\end{array}$ & 4 & 0 & 0.009792 & 0.107712 & 0.009792 \\
\hline \hline
\end{tabular}

In Table I, the minimum transmission times correspond to the best case, in which the slave can be reached by the master without repeater and the transmission is successful without retry. The maximum response times correspond to the worst case, the transmission need retry and use maximum repeater numbers to reach the destination slave. In both cases, the transmission times are greatly smaller than the time constraints of 10 seconds.

If $T_{w r}$ is configured to 154 timeslots $(\cong 1.5 / 0.009792)$ which is the maximum delay time for a response generated by the slave, we get the following table.

TABLE II

RESPONSE TIME TO A REQUEST

\begin{tabular}{ccc}
\hline \hline Channel Model & $\begin{array}{c}\text { Maximum } \\
\text { response time (s) }\end{array}$ & $\begin{array}{c}\text { Minimum } \\
\text { response time (s) }\end{array}$ \\
\hline Ring_10 & 1.66464 & 1.547136 \\
Ring_100 & 1.723392 & 1.547136 \\
RandArea Np_100 & 1.782144 & 1.547136 \\
RandArea Np_200 & 1.899648 & 1.547136 \\
\hline \hline
\end{tabular}

In Table II, we can see that the main part of the maximum and minimum response time to a request is the response generation delay, as the transmission time is small. If the network layer can get the precise $T_{w r}$ value, the response time to a request can be smaller. In current case, the response time to a request can satisfy the requirement of individual metering reading time constraint ( $<30$ seconds).
TABLE III

AVERAGE DURATION OF SFN

\begin{tabular}{cc}
\hline \hline Channel Model & Average duration (s) \\
\hline Ring_10 & 0.2996 \\
Ring_100 & 4.2096 \\
RandArea Np_100 & 4.1058 \\
RandArea Np_200 & 10.1239 \\
\hline \hline
\end{tabular}

Table III shows that the average duration is small, even 10.1239 seconds for polling 199 slaves. In 30 seconds, the master can poll all the slaves once and has residual time to implement other tasks, or poll certain slaves for several times to withdrawing more alarm information when the packet indicates that there is more alarm information stored in the slave. If the system sets a periodic task for polling all slaves once every 30 s to get the slave alarm information, the time constraint of the alarm delay in DA application, can be guaranteed.

\section{B. Timing performance in combine of $M V$ and $L V P L C$ network}

The timing performance of REMPLI PLC system should be consisted of two parts: timing performance in an autonomous MV PLC network performance and timing performance in an autonomous LV PLC network. The generic communication procedure is shown in Fig. 3.

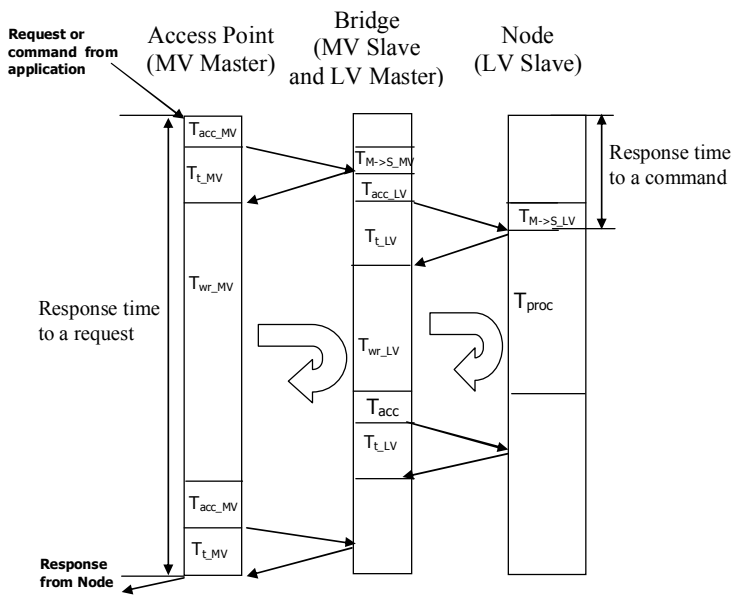

Fig. 3. Schematic representation of application-to-application delay at the Network Layer level in REMPLI PLC network

The response time $\left(T_{c}\right)$ to a command is defined as the time from the command packet ready for transmission in Access Point to the packet arriving the LV Node $i$. After the Access Point sends the command message, the Bridge receives it and checks the destination address in the packet. If the destination address is a LV Node address, which the Bridge connects with, the Bridge transfers it to its LV master part. If the LV master has been transmitting some packets, it must wait $T_{a c c_{L} L}$ for this new packet transmission. The response time to a command can be represented by the following formula.

$$
T_{c}(i)=T_{a c c_{-} M V}+T_{M \rightarrow S_{-} M V}(\text { Bridge })+T_{a c c_{-} L V}+T_{M \rightarrow S_{-} L V}(i) \text { (6) }
$$

Where the LV and MV in subscripts refer to power grids those time issues concern.

The response time $\left(T_{r}\right)$ to a request is defined as the duration from the request packet ready for transmission in 
Access Point to the Response received from Node $i$. The calculation formula is shown in (7).

$$
\begin{aligned}
& T_{r}(i)=T_{a c c \_M V}+T_{t_{-} M V}(\text { Bridge })+T_{w r_{-} M V}(i)+T_{a c c_{-} M V}+T_{t_{-} M V}(\text { Bridge }) \\
& \text { And } T_{w r_{-} M V} \text { should be }(8) . \\
& T_{w r_{-} M V}(i)>T_{a c c_{-} L V}+T_{t_{-} L V}(i)+T_{w r_{-} L V}+T_{a c c_{-} L V}+T_{t_{-} L V}(i)
\end{aligned}
$$

The other performance metrics is the average polling time for the Access Point to poll all LV Nodes to get all LV nodes data through one Bridge. The time is from sending the polling LV Node 1 message from the Access Point to receiving the data of the LV Node $n$ by the Access Point. The transmission can be concurrent in the autonomous MV PLC network and autonomous LV PLC network. It means that the Access Point sends the next polling, after it receives the confirm of last polling. At the same time, the LV master starts to poll the LV slave after receiving the polling packet from the MV slave. When a slave need send a confirm to the master after it receives a polling packet, the confirm will be combined with a data if there are one or more data which have been stored in the slave and been waiting to send back to the master. This method has maximum utilization of bandwidth. For some nodes, it still needs $T_{w r_{-} M V}$ to generate a new polling to get back the data from Bridge. $T_{w r_{-} M V}$ should be (9).

$$
T_{w r_{-} M V}(i)=T_{a c c_{-} L V}+T_{t_{-} L V}(i)
$$

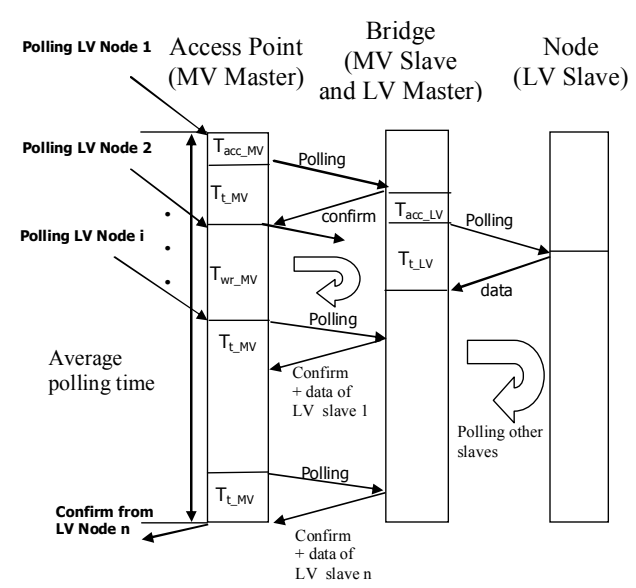

Fig. 4. Schematic representation of average polling time at the Network Layer level in REMPLI PLC network

In simulation scenarios, MV power grid is Ring_10 channel model, and the LV power grid can use all available channel Models. The MV slave part of the Bridge can be node_2 or node_6 in the MV Ring_10 channel model. From the Physical emulator, we know that node_2 always communicates with master directly and does not retry, by contraries, node_ 5 communicates with master through one or two repeater and sometimes, retry will be happened.

In the scenarios of the response time to a command and response, the $T_{a c c_{-} M V}$ and $T_{a c c \_L V}$ can be ignored, due to the same reason of the case of an autonomous PLC network. So the (8) can be simplified to (10)

$$
T_{w r_{-} M V}(i)=T_{t_{-} L V}(i)+T_{w r_{-} L V}+T_{t_{-} L V}(i)
$$

For assuring that the Access Point can get the LV slave response from the Bridge, $T_{t_{-} L V}(i)$ should be the maximum value. $T_{w r_{-} L V}$ is configured to 154 timeslots as the section $A$. So $T_{t_{-} L V}(i)$ will be different for the different LV Node $i$.

In the scenarios of the average polling time, $T_{w r_{-} M V}$ is difficult to calculate with (9), in view of variable $T_{t-L V}$ which includes possible retry. So we configure $T_{w r-M V}$ to the average polling time of the LV autonomous PLC network, for assuming that the Access Point could get the data back.

TABLE IV

RESPONSE TIME TO A COMMAND (NODE 2 AS BRIDGE)

\begin{tabular}{ccc}
\hline \multicolumn{2}{c}{ RESPONSE TIME TO A COMMAND (NODE_2 AS BRIDGE) } \\
LV segment & $\begin{array}{c}\text { Maximum } \\
\text { response time (s) }\end{array}$ & $\begin{array}{c}\text { Minimum response } \\
\text { time (s) }\end{array}$ \\
\hline Ring_10 & 0.078336 & 0.019584 \\
Ring_100 & 0.107712 & 0.019584 \\
RandArea Np_100 & 0.137088 & 0.019584 \\
RandArea Np_200 & 0.137088 & 0.019584 \\
\hline \hline & & \\
RESPONSE TIME TO A COMMAND (NoDE_5 AS BRIDGE)
\end{tabular}

Table IV and V show that simulation results of the response time to a command are smaller than $200 \mathrm{~ms}$.

TABLE VI

RESPONSE TIME TO A REQUEST (NODE 2 AS BRIDGE)

\begin{tabular}{lcc}
\hline \hline LV segment & Maximum & Minimum response \\
Channel Model & response time (s) & time (s) \\
\hline Ring_10 & 1.703808 & 1.586304 \\
Ring_100 & 1.76256 & 1.586304 \\
RandArea Np_100 & 1.821312 & 1.586304 \\
RandArea Np_200 & 1.821312 & 1.586304 \\
\hline \hline \multicolumn{3}{c}{ TABLE VII } \\
\multicolumn{3}{c}{ RESPONSE TIME TO A REQUEST (NODE_5 AS BRIDGE) } \\
\hline \hline LV segment & Maximum & Minimum response \\
Channel Model & response time (s) & time (s) \\
& & 1.703808 \\
Ring_10 & 1.821312 & 1.703808 \\
Ring_100 & 1.880064 & 1.703808 \\
RandArea Np_100 & 1.938816 & 1.703808 \\
RandArea Np_200 & 1.938816 & \\
\hline \hline
\end{tabular}

\begin{tabular}{|c|c|c|c|}
\hline $\begin{array}{l}\text { LV segment } \\
\text { Channel Model }\end{array}$ & $\begin{array}{c}T_{w r_{-} M V} \\
\text { (timeslot) }\end{array}$ & $\begin{array}{l}\text { Average duration (s) } \\
\text { (node_2 as Bridge) }\end{array}$ & $\begin{array}{c}\text { Average duration (s) } \\
\text { (node_5 as Bridge) }\end{array}$ \\
\hline Ring 10 & 28 & 0.871488 & 0.470016 \\
\hline Ring 100 & 418 & 10.08576 & 6.051456 \\
\hline RandArea Np 100 & 379 & 9.792 & 5.67936 \\
\hline RandArea $\mathrm{Np}_{2} 200$ & 983 & 21.5424 & 13.542336 \\
\hline
\end{tabular}

Table VI and VII show that the maximum response time is smaller than 2 seconds.

TABLE VIII

AVERAGE DURATION

Table VIII shows that the maximum average polling is small than 30 seconds.

So in both an autonomous PLC network and the total REMPLI PLC network, the simulation results show that the REMPLI PLC system can satisfy the time requirements of DA/DSM applications.

\section{New Scheduling Policy}

In a multitasking environment, applications should be 
served differently in order to satisfy their different QoS requirements. In the REMPLI system, the dispatcher within the network layer of the master provides a QoS mechanism by permitting an optimal share of the network bandwidth among different traffic generated by the applications. The dispatcher function is called when the medium is currently free and can be used for a new transmission in the next timeslot. Thus, the dispatcher must decide, at the master side, which is the next packet to be sent, among the different available packets, and based on the different QoS requirements.

The Network Layer provides three priority levels: 0,1 and 2 for aperiodic task and hard and soft levels for the periodic polling. The order in which the dispatcher inspects the structure is given in Fig. 5.

1 - Aperiodic Requests with priority 0

2 - Hard Periodic Polling

3 - Aperiodic Requests with priority 1

4 - Soft Periodic Polling

5 - Aperiodic Requests with priority 2

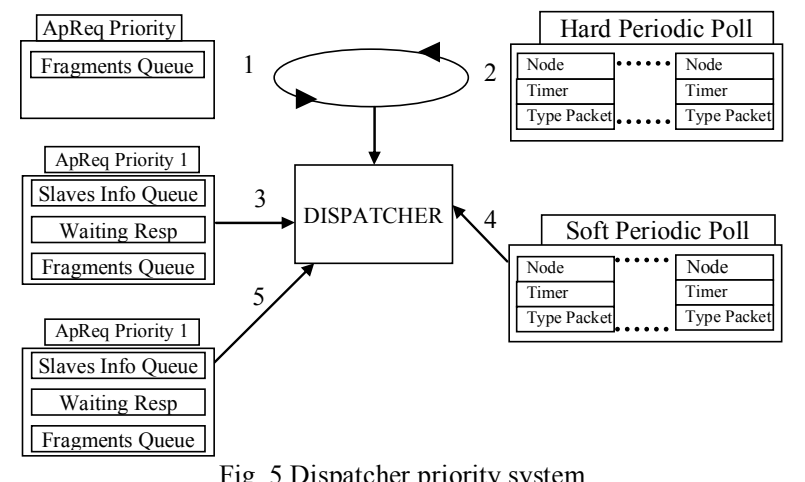

A Round-Robin mechanism exists between aperiodic packets of priority 0 and hard periodic packets. This allows to maintain a correct management of the network (through the hard periodic polling) for one part, and to allow critical aperiodic packets to immediately be transmitted for another part; without creating network monopolization by any of them.

A dual-priority scheduling policy is used between the aperiodic request and periodic polling. Periodic packets possess two levels of priority: low and high level, whilst aperiodic packets are scheduled using a medium priority level. According to this, periodic packets can run immediately at a low level while there is no aperiodic traffic. Otherwise, a soft periodic task should only be sent when it is promoted into a hard periodic packet in order to guarantee the completion of the current activation by dual-priority dispatcher with deadline relaxation [8]. Deadline relaxation principle is to relax deadline constraint of soft periodic polling, permit missing deadline of soft periodic polling, and make its deadline as the promotion time. It is possible to build a simpler and faster dispatcher, based on the Dual-Priority policy and still respecting the most important constraint in our system: the periodicity.
TABLE IX

PACKET TYPES MAPPING TO PRIORITY

\begin{tabular}{|c|c|c|}
\hline Priority Level & Packet Type & \\
\hline Aperiodic Requests Priority 0 & $\begin{array}{l}\text { Urgent Commands } \\
\text { application priority) }\end{array}$ & $\overline{\text { (highest }}$ \\
\hline Hard Periodic Polling & $\begin{array}{l}\text { Application } \\
\text { application) }\end{array}$ & (periodic \\
\hline Aperiodic Requests Priority 1 & $\begin{array}{l}\text { Application packets } \\
\text { application priority) }\end{array}$ & (medium \\
\hline Soft Periodic Polling & \multirow{2}{*}{\multicolumn{2}{|c|}{$\begin{array}{l}\text { Network management service } \\
\text { Application packets (low application } \\
\text { priority) }\end{array}$}} \\
\hline Aperiodic Requests Priority 2 & & \\
\hline
\end{tabular}

Table IX shows the QoS mapping between the packet type and the network priority.

In [8], [9], the simulation results have proved that this new scheduling policy specifies the order of transmitting packets and supports QoS requirements.

\section{CONCLUSION}

In this paper, we proposed an embedded powerline communication in the DA/DSM RTUs to construct a cost-effective, high speed communication network for DA/DSM application. We analyzed and simulated the REMPLI system to evaluate the communication performance. Moreover a new scheduling policy is proposed to provide the applications with differentiated QoS in a multitasking environment. From those analysis and simulation results, we can make a conclusion that REMPLI PLC system can meet the demands of DA/DSM application.

\section{REFERENCES}

[1] Sioe Mak, Denny Radford, "Communication System Requirements for Implementation of Large Scale Demand Side Management and Distribution Automation", IEEE Transactions on Power Delivery, Vol. 11, No. 2, April 1996

[2] M. Ostertag, Ch. Imboden, "High Data Rate, Medium Voltage Powerline Communications for Hybrid DA/DSM", In Proceedings of the IEEE Power Engineering Society Transmission and Distribution Conference, v 1, 1999, p 240-245

[3] Gerd Bumiller, "Single Frequency Network Technology for Medium Access and Network Management," 6th International Symposium on Power-Line Communications and its applications, March 2002

[4] Gerd Bumiller, "Power-Line Physical Layer Emulator for Protocol Development", In 8th International Symposium on Power-Line Communications and its applications.

[5] Deliverable 1.6 "QoS Requirements Analysis", www.rempli.org

[6] Deliverable 1.1 "Application Requirements Report”, www.rempli.org

[7] Gerd Bumiller, Liping Lu, Yeqiong Song, "Analytic Performance Comparison of Routing Protocols in Master-Slave PLC Networks", In 9th International Symposium on Power-Line Communications and Its Applications

[8] Raul Brito and Yeqiong Song, "A Dispatching Mechanism Providing REMPLI Applications with QoS", In 10th IEEE International Conference on Emerging Technologies and Factory Automation.

[9] Liping Lu, Raul Brito, Yeqiong Song, "QoS and Performance of REMPLI PLC Network", In 1st Workshop on Networked Control System and Fault Tolerant Control - NeCST Workshop 2005 